\title{
Association Between Chronic Rhinosinusitis and The Vestibular System
}

\author{
Kadri Ila ${ }^{1,2,}$ - Emre Soylemez ${ }^{1,}$ - Nihat Yilmaz ${ }^{1,}$ - Adrien A Eshraghi ${ }^{2}$ \\ 1 Department of Otolaryngology, Faculty of Medicine, Karabuk University. Karabuk/Turkey \\ 2 Department of Otolaryngology, University of Miami Miller School of Medicine. Florida/USA
}

Kadri Ila; Assistant Professor. Department of Otolaryngology, Faculty of Medicine, Karabuk University. Orcid: 0000-0003-3211-6107

Emre Soylemez; Audiology. Department of Otolaryngology, Faculty of Medicine, Karabuk University. Orcid: 0000-0002-7554-3048

Nihat Yilmaz; Assistant Professor. Department of Otolaryngology, Faculty of Medicine, Karabuk University. Orcid: 0000-0003-1575-1280 Adrien A Eshraghi; MD, MSc, FACS Professor of Otolaryngology. Department of Otolaryngology, University of Miami Miller School of Medicine. Orcid: 0000-0002-4083-905X

\begin{abstract}
Objective: Objective: Chronic rhinosinusitis (CRS) is inflammation of the nasal mucosa and paranasal sinuses. Subjective complaint of dizziness can be observed in patients with CRS. However, a review of the literature did not reveal any prospective studies that have used videonystagmography (VNG) to examine the vestibular system of patients with CRS. The goal of this study is the evaluation of the vestibular system of CRS patients.

Methods: The study comprised 30 patients with CRS and 30 control subjects. The vestibular system was evaluated through VNG. The association with vestibular function was evaluated according to the Lund-Mackay score, the Sino-Nasal Outcome Test-22 (SNOT-22) score, and the existence of nasal polyps.
\end{abstract}

Results: Abnormal caloric test results were observed in eight $(26.6 \%)$ of the 30 CRS patients. The caloric test results were normal for the control group. The oculomotor test results with VNG were normal for the CRS and control groups. The abnormal caloric results were higher for the CRS with nasal polyp patients than for the CRS without nasal polyp patients. The VNG showed no correlation among the SNOT-22 scores and the Lund-Mackay scores in patients with CRS.

Conclusion: Abnormal caloric responses can be observed in patients with CRS with nasal polyps.

Keywords: Sinusitis, vestibular function tests, peripheral vestibular diseases.
Correspondence: Kadri Ila MD,

Assistant Professor of Otolaryngology, Karabuk University Faculty of Medicine e-mail: dr.kila@gmail.com

Received: 04.07.2019; Accepted: 22.07.2019

Online available at: www.entupdates.org

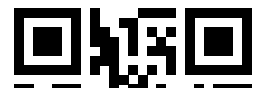
隹 茴被 


\section{Introduction}

Chronic rhinosinusitis (CRS) is inflammation of the nasal mucosa and paranasal sinuses. The European Position Paper on Rhinosinusitis and Nasal Polyps (EPOS) has defined CRS as the presence of two or more symptoms, one of which is either nasal blockage, obstruction, or congestion or nasal discharge (anterior or posterior nasal drip) in addition to facial pain or pressure, reduction or loss of smell, and inflammation that should be observable through endoscopy or computed tomography (CT) after more than 12 weeks. ${ }^{[1]}$ Patients with CRS with nasal polyps (CRSwNPs) complain mostly of nasal obstruction and loss of smell, and patients with CRS without nasal polyps (CRSsNPs) have mainly facial pain and nasal discharge. ${ }^{[2]}$ The prevalence of CRS is $13 \%$ in the United States and $11 \%$ in Europe. CRSsNPs was more common than CRSwNPs. ${ }^{[3]}$

The vestibular system consists of the sensory organs of the inner ear (utricle, saccule, and semicircular canals) and connections to the central nervous system (brain stem, cerebellum, and cerebral cortex), ocular system, and postural muscles. ${ }^{[4]}$ Vestibular tests, such as the Romberg test, the head thrust test, the Fukuda stepping test, posturography, and videonystagmography (VNG) are often used to evaluate the vestibular system. VNG consists of oculomotor (saccades assessment, smooth pursuit, and optokinetic) and caloric tests. $^{[5]}$

Subjective complaints of dizziness can be seen in patients with CRS; there are the inclusion of items on dizziness in some questionnaires, such as the Sino-Nasal Outcome Test-22 (SNOT-22) ${ }^{[6]}$ Few studies have investigated the association between CRS and vertigo. ${ }^{[7-9]}$ The mechanisms behind the relationship between vestibular function and rhinosinusitis have not yet been elucidated. A possible mechanism is the stimulation of the trigeminal nerve may innervate the auditory and vestibular portions of the labyrinth. ${ }^{[10]}$ To date, no prospective studies have used VNG to examine vestibular function in patients with CRS. The aim of this study was the evaluation of vestibular function in patients with CRS.

\section{Methods}

\section{Subjects and Data Collection}

A total of 30 patients with CRS and 30 subjects without CRS as the control group were included in this study. The Institutional Review Board approved the protocol for this study, and informed consent was obtained from each participant. Patients with ear problems, such as tympanic membrane perforation, chronic otitis media, and serous otitis media, patients with vertigo, patients with visual problems as indicated by an oculomotor test, and patients with hearing loss $>20 \mathrm{~dB}$ were excluded from the study. Pure tone audiometry was performed to all participants at 250-8000 Hz frequency (AC 40, DK-500; Interacoustics, Middelfart, Denmark).

\section{Evaluation of Chronic Rhinosinusitis Patients}

CRS was diagnosed in accordance with the EPOS definition. ${ }^{[1]}$ Nasal endoscopy was performed in all patients. Paranasal sinus computed tomography (PNS CT) was performed in all patients with chronic sinusitis. The Lund-Mackay (LM) score was used for computed tomography (CT) evaluation in CRS patients. Each sinus (maxillary, frontal, anterior ethmoid, posterior ethmoid, and sphenoid) was scored as follows: 0 , no opacification; 1, partial opacification; or 2, complete opacification. The ostiomeatal complex was scored as follows: 0 , no opacification; or 2, opacification. Each side was scored separately, 0-12, and the overall CT score was $0-24{ }^{[1]}$ The correlation between the LM score and vestibular functions was evaluated.

The Sino-Nasal Outcome Test-22 (SNOT-22) questionnaire consists of 22 questions. Each question is rated $0-5$ ( 0 , no problem to 5 , as bad as possible). The total score $(0-110$, from best to worst quality of life) is obtained by adding the scores for the questions. ${ }^{[6]}$ The correlation between the SNOT-22 score and vestibular functions was assessed.

The patients with CRS were divided into two groups: those with CRSwNPs and those with CRSsNPs. The patients with CRSwNPs and those with CRSsNPs were compared in terms of vestibular function.

\section{Evaluation of Vestibular Function}

The VNG, including oculomotor (saccade, smooth pursuit test, and optokinetic test) and caloric tests, was performed with a videonystagmographic system (Spectrum software; Micromedical Technologies, Chatham, IL, USA). When caloric testing was performed, each ear was stimulated separately by $47^{\circ} \mathrm{C}$ hot air and $27^{\circ} \mathrm{C}$ cold air for 60 seconds. A unilateral abnormal caloric response was deemed to be more than $25 \%$ asymmetry between the two ears. A bilateral abnormal caloric response was considered when the total 
slow phase velocity was lower than $12 \%$ with stimulation with hot air and cold air for both ears. The accuracy, velocity, and latency values were recorded for the saccade test. A value of $80-134 \%$ for accuracy was considered normal. A value less than $275 \%$ for velocity was considered to be pathological, and a value higher than $260 \mathrm{~ms}$ for latency was considered pathological. Gain and asymmetry were recorded for the smooth pursuit test. Gain values less than 0.75 at $1 \mathrm{kHz}, 0.80$ at $2 \mathrm{kHz}$, or 0.75 at $4 \mathrm{kHz}$ were considered pathological. The gain value was evaluated for the optokinetic test, and a gain value less than $0.6 \%$ was considered to pathological.

\section{Statistical Analysis}

The statistical analysis was performed using SPSS 19.0 (SPSS software, SPSS Inc., Chicago, IL, USA). The distributional properties of the variables were evaluated through the Shapiro-Wilk test. The descriptive statistics were expressed as median (minimum-maximum), mean \pm standard deviation (SD), and frequency (\%). The chi-square test was used to evaluate the independence of the categorical variables. It was also used to evaluate the relationships between the groups and gender. The independence of the categorical variables for small sample sizes was evaluated through Fisher's exact test. The odds ratio was used to evaluate the caloric test results of the CRS patients and those in the control group. Binary group comparisons were performed by use of the Student's t-test for normally distributed continuous variables. The Mann-Whitney U test was used for the non-normally distributed continuous variables. A $p$ value of $<0.05$ was considered statistically significant.

\section{Results}

\section{Clinical Characteristics of Subjects}

Of the 30 patients with sinusitis, $16(53.3 \%)$ were female, and $14(46.6 \%)$ were male. The mean age was $38.20 \pm 13.83$. Of the 30 patients in the control group, 20 (66.7\%) were female, and $10(33.3 \%)$ were male. The mean age was $43.73 \pm 14.62$. There was no significant difference between the groups in terms of gender and age ( $>0.05)$.

\section{Vestibular Function in Patients with Chronic Rhinosinusitis}

Abnormal caloric test results were obtained for eight (26.6\%) of the 30 patients with sinusitis. The caloric test results were normal for the control group. The abnormal caloric results were higher for the CRS patients than for the control group $(\mathrm{p}<0.05$; odds ratio 23.04 [95\% confidence interval 1.26-420.39]). There was no significant difference between the control group and the CRS group in terms of saccade accuracy, saccade latency, smooth pursuit asymmetry, smooth pursuit gain values, or optokinetic gain values $(p>0.05)$ (Table 1$)$.

\begin{tabular}{|c|c|c|c|}
\hline & CRS group & Control group & $p$ value \\
\hline $\begin{array}{l}\text { Abnormal } \\
\text { caloric test (n) }\end{array}$ & $8 / 33$ & $0 / 30$ & $\mathrm{p}<0.05^{a}$ \\
\hline \multicolumn{4}{|l|}{ Saccade } \\
\hline Accuracy & $92.43 \pm 3.95$ & $93.25 \pm 4.36$ & $p>0.05^{b}$ \\
\hline Latency & $150.47 \pm 34.67$ & $145.67 \pm 35.41$ & $p>0.05^{b}$ \\
\hline \multicolumn{4}{|l|}{ Smooth Pursuit } \\
\hline Gain & $0.95(0.65-1.00)$ & $0.97(0.75-1.00)$ & $p>0.05^{c}$ \\
\hline Asymmetry & $5.50(0-57)$ & $4.50(0-54)$ & $p>0.05^{c}$ \\
\hline \multicolumn{4}{|l|}{ Optokinetic } \\
\hline Gain & $0.83 \pm 0.12$ & $0.88(0.60-1.00)$ & $p>0.05^{c}$ \\
\hline
\end{tabular}

a Chi square, Odds ratio

${ }^{\mathrm{b}}$ Student t-test, Mean \pm SD

'Mann Whitney-U test, Median (min-max)

\section{Relationships among Sino-Nasal Outcome Test-22 and Lund-Mackay Scores and Videonys- tagmography Results}

The mean SNOT-22 score for the CRS patients was $51.83 \pm 25.60$. The mean SNOT-22 score for the patients with abnormal caloric test results was $46.17 \pm 27.09$, and for the patients with normal caloric test results, it was $53.25 \pm 25.62$. No significant correlation was found between the SNOT-22 scores and caloric test abnormality $(\mathrm{p}>0.05)$. No difference was found in the SNOT-22 scores in terms of saccade accuracy, saccade latency, smooth pursuit asymmetry, smooth pursuit gain values, and optokinetic gain values in patients with CRS ( $p>0.05)$.

The mean LM scores for the patients with CRS was $13.83 \pm 6.24$. The mean LM scores for the patients with abnormal caloric test results was $18.17 \pm 5.34$, and for the patients with normal caloric test results, it was $12.75 \pm 6.07$. No significant correlation was found between the LM scores 
and caloric abnormality in the CRS patients ( $>0.05$ ). There was no difference in the LM scores in terms of saccade accuracy, saccade latency, smooth pursuit asymmetry, smooth pursuit gain values, and optokinetic gain values in patients with CRS ( $>>0.05)$.

\section{Relationship Between Nasal Polyps and Vide- onystagmography Results}

Fourteen (46.6\%) of 30 patients had CRSwNPs, and 16 $(53.3 \%)$ had CRSsNPs. Seven (50\%) of 14 patients with CRSwNPs had abnormal caloric test results, and one (6.25\%) of 16 patients with CRSsNPs had abnormal caloric test results. The abnormal caloric results were higher for the CRSwNPs patients than for the CRSsNPs group $(p<0.05$; odds ratio 15.00 [95\% confidence interval $1.53-$ 146.55]). There was no difference between the CRSwNPs and CRSsNPs groups in terms of saccade accuracy, saccade latency, smooth pursuit asymmetry, smooth pursuit gain values, and optokinetic gain values $(\mathrm{p}>0.05)$.

\section{Discussion}

Patients with acute or chronic sinusitis often complain of having also dizziness, a condition that is defined as sinugenic vertigo in the literature. ${ }^{[12]}$ Watson-Williams presented the first case report of sinugenic vertigo. The patient had presented with recurrent vertigo attacks that were resolved after sinus surgery. ${ }^{[13]}$ Few studies have been conducted since. Haid reported on a sinugenic vertigo diagnosis for 30 of 4.112 vertigo patients. Fifteen of these 30 patients were reported to have followed up. Fourteen patients reported no complaint at the end of sinus treatment. ${ }^{[12]}$

Gavriel et al included 16 patients with a rhinosinusitis diagnosis in their study. Eleven had acute rhinosinusitis, two had subacute rhinosinusitis, and three had recurrent rhinosinusitis. Computerized dynamic posturography (CDP) was performed for all the patients. The results for 13 patients were normal, and for 3 patients were pathological. After treatment for rhinosinusitis, the follow-up CPD results were normal for two of the three patients, and the other patient showed considerable improvement. ${ }^{[7]}$

A study of 52 patients with unilateral benign paroxysmal positional vertigo (BPPV) and 46 normal subjects found a higher frequency of sinus disease in the BPPV patients than in the control group. ${ }^{[8]}$ Brody-Camp et al examined a database of 3.078 patients who had undergone VNG over an eight-year period. The study included 70 patients with a sinusitis diagnosis. The VNG results indicated abnormal for 35 (50\%) of 70 patients with CRS and 1.477 (49.1\%) of 3.008 patients without CRS. The limitation was the study's retrospective design. ${ }^{[9]}$ In our study, abnormal caloric test result was observed in $50 \%$ of CRSwNPs patients and in $6.25 \%$ of CRSsNPs patients. In a study on vestibular neuritis, Coats et al found a higher frequency (19.1\%) of sinusitis in patients with multiple attacks than those with only a single attack or the subjects in the control group. The study suggest that vestibular neuritis could be associated with sinusitis. ${ }^{[14]}$

Hung et al examined a database of 3325 patients with sudden sensorineural hearing loss and 9975 subject for control group. The study demonstrated that sudden SNHL is associated with chronic rhinosinusitis. A possible mechanism stated that infections in the sinuses can contribute to the inner ear via eustachian tube and inflammatory processes could damage the inner ear ${ }^{[15]}$ Lin et al included 82 patients with chronic rhinosinusitis and 21 control subject in their study. The study showed that sensorineural hearing loss was significantly higher than the control group. In the study, the possible mechanism stated that, the pathogens and inflammation of the paranasal sinus could be migrate through the Eustachian tube to the inner ear. ${ }^{[16]}$

The exact mechanism of the association between vestibular function and CRS is not known. Trigeminal nerve can be stimulated in chronic rhinosinusitis as trigeminal nerve endings are distributed throughout the nasal mucosa. ${ }^{[17]}$ Poletti et al investigated trigeminal sensitivity in CRSwNPs. They found that CRSwNPs patients had a significantly higher trigeminal threshold than the healthy control group. ${ }^{[18]}$ The trigeminal ganglion stimulations may innervate the auditory and vestibular portions of the labyrinth. ${ }^{[10]}$ Vass et al applied iontophoretic injections of biocytin to the trigeminal ganglion in guinea pigs. Labeled fibers were observed in the cochlea and the vestibular labyrinth 24 hours later. ${ }^{[19]}$ The activation of the trigeminal vascular system (TVS), which consists of the trigeminal nuclei, ganglion, and nerve, can cause peripheral vestibular dysfunction. ${ }^{[20]}$

In the present study, $24.2 \%$ of patients with CRS had abnormal caloric test results; however, all the oculomotor test results were normal. The SNOT-22, which is a modified form of the SNOT-20, is a widely used validated questionnaire to assess the quality of life of CRS patients. ${ }^{\left[{ }^{[6]}\right.}$ This study found no correlation between the SNOT-22 scores and vestibular functions. The LM score was used to evaluate the degree of CRS through evaluations of parana- 
sal sinus CT. ${ }^{[1]}$ No correlation was found between the LM scores and vestibular functions. CRSwNP and CRSsNP were reported to have different inflammatory patterns. Patients with CRSwNP were found to have a better response to nasal and systemic corticosteroids than those with CRSsNP. ${ }^{[2]}$ In the current study, we found that abnormal caloric results of the CRSwNPs were higher than the CRSsNPs group. The mechanism has not been fully elucidated. Trigeminal stimulation may affected vestibular system or it may be the result of vestibular damage caused by inflammation through the eustachian tube.

The limitation of this study was that vestibular evoked myogenic potentials (VEMPs) did not performed to evaluate functions of otolith organs such as the saccule and the

\section{References}

1. Fokkens WJ, Lund VJ, Mullol J, et al EPOS 2012: European position paper on rhinosinusitis and nasal polyps 2012. A summary for otorhinolaryngologists. Rhinology 2012;50:1-12.

2. Dietz de Loos DA, Hopkins C, Fokkens WJ. Symptoms in chronic rhinosinusitis with and without nasal polyps. Laryngoscope 2013;123:57-63.

3. Cho SH, Kim DW, Gevaert P. Chronic Rhinosinusitis without Nasal Polyps. J Allergy Clin Immunol Pract 2016;4:575-82.

4. Mucha A, Collins MW, Elbin RJ, et al A Brief Vestibular/Ocular Motor Screening (VOMS) assessment to evaluate concussions: preliminary findings. Am J Sports Med 2014;42:2479-86.

5. Zamysłowska-Szmytke E, Szostek-Rogula S, Śliwińska-Kowalska M. Bedside examination for vestibular screening in occupational medicine. Int J Occup Med Environ Health 2015;28:379-87.

6. Hancı D, Altun H, Şahin E, Altıntoprak N, Cingi C. Turkish translation, cross-cultural adaptation and validation of the SinoNasal Outcome Test (SNOT)-22. ENT Updates 2015;5:51-7.

7. Gavriel H, Shlamkovitch N, Kessler A, Eviatar E. Role of computerized dynamic posturography in evaluating sinugenic vertigo. Ann Otol Rhinol Laryngol 2013;122:263-8.

8. Cohen HS, Stewart MG, Brissett AE, Olson KL, Takashima M, Sangi-Haghpeykar H. Frequency of sinus disease in normal subjects and patients with benign paroxysmal positional vertigo. ORL J Otorhinolaryngol Relat Spec 2010;72:63-7.

9. Brody-Camp S, Risey JA Jr, McCoul ED. Vestibular Characteristics of Patients with Chronic Rhinosinusitis. OTO Open 2018;2:1-3. utricle in patients with CRS. And also a number of studies are needed to fully understand the mechanism between CRS and the vestibular system.

\section{Conclusions}

This study demonstrated that abnormal caloric responses can be observed in patients with CRSwNP. No correlations were found among the SNOT-22 and LM scores and vestibular function.

\section{Acknowledgements}

There is no conflict of interest. The authors are responsible for the content and writing of this paper.

10. Shore SE, Vass Z, Wys NL, Altschuler RA. Trigeminal ganglion innervates the auditory brainstem. J Comp Neurol 2000;419:271-85.

11. Lund VJ, Mackay IS. Staging in rhinosinusitus. Rhinology 1993;31:183-4.

12. Haid T. Vertigo originating from inflammation of the paranasal sinuses (the so-called sinugenic vertigo). Adv Otorhinolaryngol 1981;27:190-7.

13. Watson-Williams P. Case of Labyrinthine Vertigo due to Sphenoidal and Ethmoidal Sinusitis. Proc R Soc Med 1924;17:95-6.

14. Coats AC. Section I: Definition of Vestibular Neuronitis. Acta OtoLaryngologica 1969;67:7-17.

15- Hung SH, Lin HC, Kao LT, Wu CS, Chung SD. Sudden sensorineural hearing loss is associated with chronic rhinosinusitis: population-based study. J Laryngol Otol 2016;130:521-5.

16- Lin X, Shan X, Lin S, Shu B, Wang Y, Xiao W. Is Sensorineural Hearing Loss Related to Chronic Rhinosinusitis Caused by Outer Hair Cell Injury? Med Sci Monit 2019;25:627-36.

17. Saliba J, Fnais N, Tomaszewski M, et al The role of trigeminal function in the sensation of nasal obstruction in chronic rhinosinusitis. Laryngoscope 2016;126:174-8.

18. Poletti SC, Cuevas M, Weile S, Hummel T. Trigeminal sensitivity in chronic rhinosinusitis: topographical differences and the effect of surgery. Rhinology 2017;55:70-4.

19. Vass Z, Shore SE, Nuttall AL, Miller JM. Direct evidence of trigeminal innervation of the cochlear blood vessels. Neuroscience 1998;84:559-67.

20. Liu YF, Xu H. The Intimate Relationship between Vestibular Migraine and Meniere Disease: A Review of Pathogenesis and Presentation. Behav Neurol 2016;2016:3182735.

This is an open access article distributed under the terms of the Creative Commons Attribution-NonCommercial-NoDerivs 3.0 Unported (CC BY- NC-ND3.0) Licence (http://creativecommons.org/licenses/by-nc-nd/3.0/) which permits unrestricted noncommercial use, distribution, and reproduc- tion in any medium, provided the original work is properly cited.

Please cite this article as: Ila K, Soylemez E, Yilmaz N, Eshraghi AA. Association Between Chronic Rhinosinusitis and The Vestibular System. ENT Updates 2019;9(2): 133-137. 